\title{
Determination of the effects 3D Margins around the treatment volume in radiotherapy planning
}

\author{
Zyad A.Tawfik*, Mohamed El-Azab Farid**, Ahmed A. Hussein**, \\ Khaled M. Elshahat*** \\ *Assiut Military Center for Radiotherapy, Assiut, Egypt \\ **Physics Department, Faculty of Science, Assiut University, Assiut, Egypt \\ ***Radiation Oncology Department, Faculty of Medicine, Al-Azhar University, Cairo, Egypt
}

\begin{abstract}
In radiotherapy treatment there are many potential error sources, which can increase the uncertainty in the dose delivery to a patient to unacceptable values. Potential error sources can be divided into four categories $^{(1,2)} ;$ (1) errors in the data transfer from treatment planning system (TPS) to treatment equipment, (2) errors in the functioning of the treatment equipment, (3) errors that that may arise from organ motion and (4) inaccuracies during the treatment planning process. Various solutions can be chosen to detect and correct some of these four errors categories, but few solutions can be utilized to detect errors in all four categories. Several margin 'recipes' are described to calculate the CTV-PTV margin $^{(5,6)}$. Once the target volume is accurately delineated, treatment fields can be defined and the distribution of the delivered dose can be calculated, optimized with utmost minimization of doses to surrounding healthy tissues. Management of organ motion has become most difficult challenges in radiation oncology. Anatomic models that describe geometric changes in organs over time offer a unique opportunity to tackle various issues related to dose planning , monitoring and to radiation delivery. To account for temporal and spatial anatomic changes during radiation treatment, real means to specify organ and/or tumor motion are required.This work aims to evaluate the safety margins for all planning tumor volume inside the radiation beam taking into consideration the possible movements of organs adjacent to the tumor location, e.g rectum and the urinary bladder. Results: Mean 3D displacement was $7.3 \mathrm{~mm}(S D, 4.4 \mathrm{~mm})$ and $7.6 \mathrm{~mm}(S D, 4.2 \mathrm{~mm})$ for laser and port film, respectively. Simulated setup with the EPID mean displacement of $3.2 \mathrm{~mm}(S D, 1.2 \mathrm{~mm})$. Distance analysis resulted in mean distances of $5.1 \mathrm{~mm}(S D, 4.9 \mathrm{~mm}), 4.3 \mathrm{~mm}(S D, 5.6 \mathrm{~mm})$, and $1.6 \mathrm{~mm}(S D, 2.4 \mathrm{~mm})$ for laser, port film, and EPID , respectively. Bladder and Rectum motion at isocenter were less than $5 \mathrm{~mm}$, with a mean of $4.5 \mathrm{~mm}$ $(S D, 1.1 \mathrm{~mm})$. In comparison with different studies were done the average margins about $12 \mathrm{~mm}$ in agreement with current study. Conclusions: Prostate displacement due effect of bladder and rectum varied within $5 \mathrm{~mm}$ to $12 \mathrm{~mm}$, the variation of displacement in average $5 \mathrm{~mm}$.
\end{abstract}

\section{Introduction}

In high dose-high precision radiotherapy era, treatment verification is necessary to assure the correct dose delivery to a patient. Several authors have proposed accuracy requirements. For instance, Shen et al 2003. [1] Proposed an accuracy requirement in absorbed dose delivery at the dose specification point of $3.5 \%$ (1 SD) for external beam treatments based on the steepness of dose-effect curves for local tumor control and normal tissue damage. For specific high dose-high precision treatments this number may be decreased, or for less demanding situations increased.

There are many potential error sources in a radiotherapy treatment, which can increase the uncertainty in the dose delivery to a patient to unacceptable values. Potential error sources can be subdivided into four categories [2]: (1) errors in the data transfer from treatment planning system (TPS) to treatment equipment, (2) errors in the functioning of the treatment equipment, and errors that are patient related due to (3) set-up errors or organ motion and (4) inaccuracies during the treatment planning process Various solutions can be chosen to detect and correct errors in these four categories but there are few solutions that can be utilized to detect errors in all four categories.

One of the most important critical steps in the radiation treatment process is the definition of the tumor and surrounding tissues. Currently a CT scan is widely used to delineate the gross tumor volume (GTV), which consists of all clinically macroscopic disease, including what is visible on imaging modalities 3 , 4. Safety margins are added to this volume to prevent geographical misses. The clinical target volume (CTV) is created by adding a certain margin to account for microscopic disease. After accurate definition of this GTV and CTV, geometric uncertainties like set-up errors and tumor motion are covered by an additional safety margin to create the planning target volume (PTV). 
These geometrical errors can be divided into systematic (every fraction the same) and random (varying from fraction to fraction) deviations from the planned situation. Several margin 'recipes' are described in literature to calculate the CTV-PTV margin 5-6. Once the target volume is accurately delineated, treatment fields can be defined and the dose distribution can be calculated and optimized

\section{Material and methods:}

Materials : 15 patients during the period May 2012 to April 2013 Linear Accelerator DMX (Varian), with photon energies 6 and 15 MV Treatment planning System, Eclipse .Computed tomography (GE) Coherence software for CT-simulator. Gammex movable laser system., Isoline QA device. Portal imaging, QA Kits for Planning system and Portal imaging.

- Method: 15 patients during the period May 2012 to April 2013 Routine pretreatment verification and individual patient dosimetry. All procedures are based on First CT Fusion images in different condition for Patient- Definition of the Target Volume in addition Safety Margin and relation between TV and surrounding organs (Risk Organs)- Final Step electronic portal imager dosimetry (EPID) as check final step verification system in treatment. Check, all system for treatment for optimize the PTV and OAR delineation margins and error in system for imaging and planning.
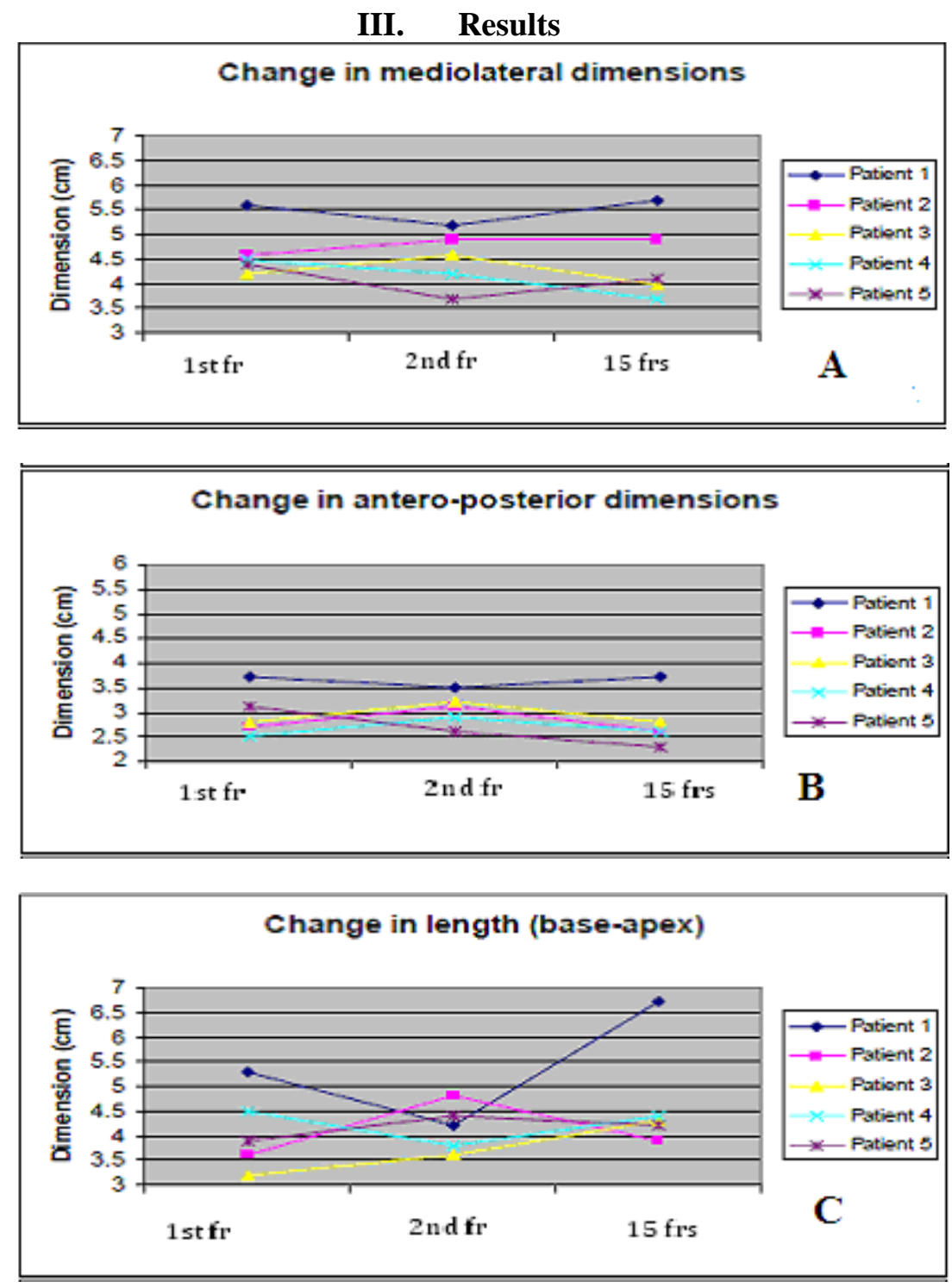


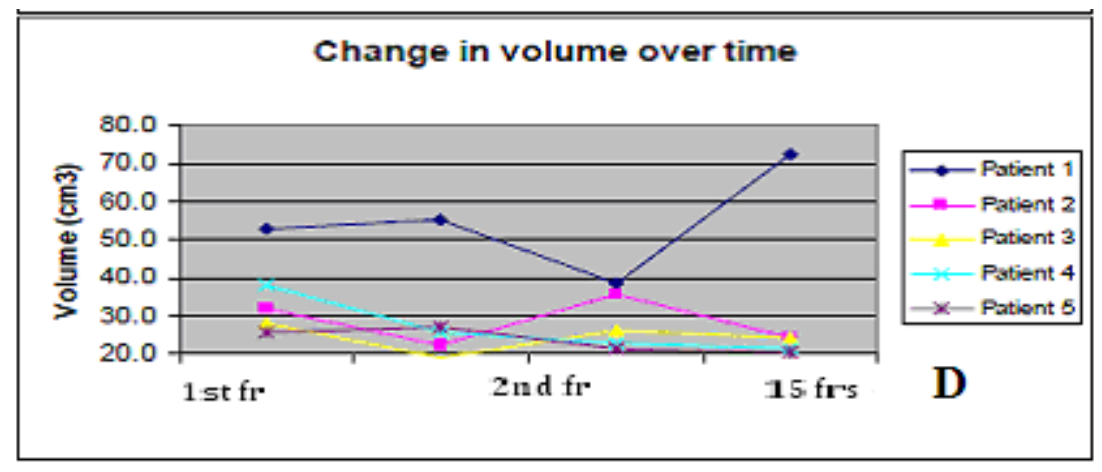

Figures (1). Changes in prostate dimensions over time. Subsequent measurements were made using the ellipsoid approximation. Change in antero-posterior dimensions -in first fraction and second fraction and change after 15 fractions. For 5 selected patients

Fig. 1: illustrate the change in PTV volume during the treatment fraction due to change in shape of prostate - the variation according the CT done for 4 selected patients in average $0.65 \mathrm{~mm}$ mediolateral direction, average $0.37 \mathrm{~mm}$ in anterior posterior direction, average $0.57 \mathrm{~mm}$ in length of Apex for prostate and all average change in volume overage $0.53 \mathrm{~mm}$ for all directions. For this results the margin in GTV to CTV should be taken about $0.5 \mathrm{~mm}$ for encompass the CTV all over radiation fields

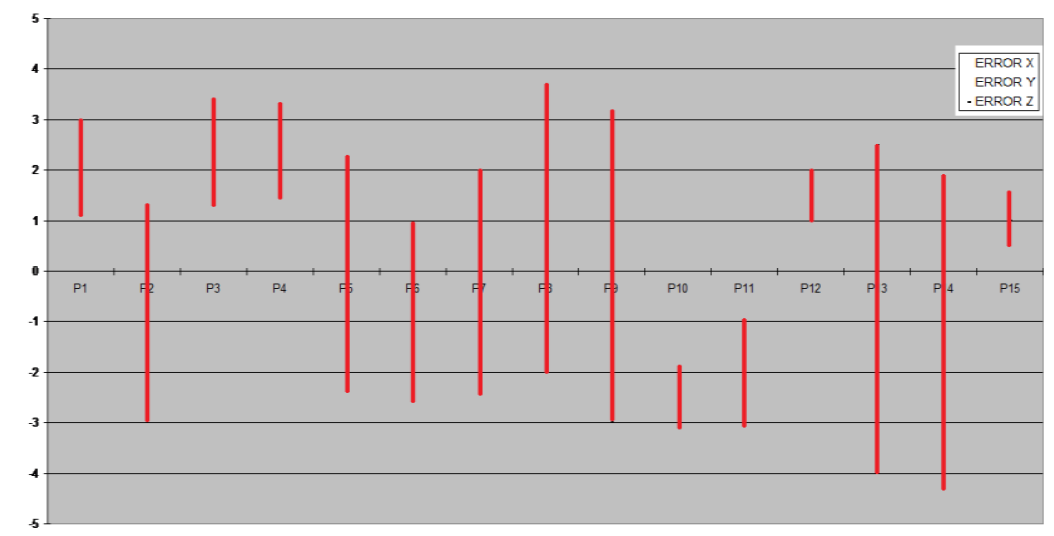

Fig( 2) Inter-fractional setup errors in $\mathrm{x}, \mathrm{y}, \mathrm{z}$ direction of 15 prostate patients, based on bony anatomy calculated by the rigid correlation matcher. For each patient, the mean translational error and its standard deviation is plotted.

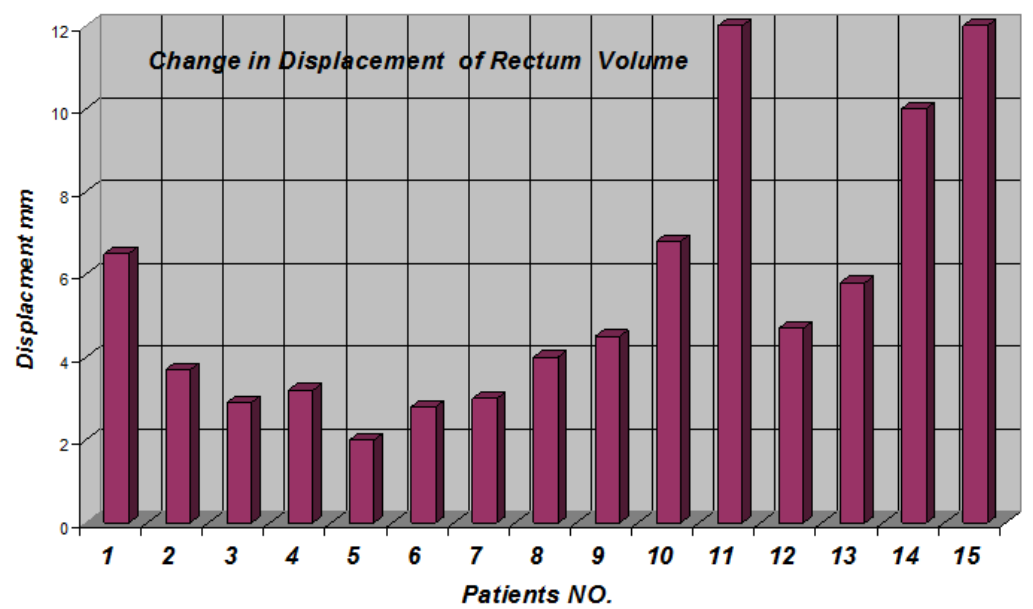

Fig (3): Displacement of Rectum Volumes

- As Show in Fig (3) : the max change in rectum volumes in patients involve this study about $12 \mathrm{~mm}$ as average in all directions .

- There is significant increase in rectum dose about 12 Gy more than stable condition for rectum. 


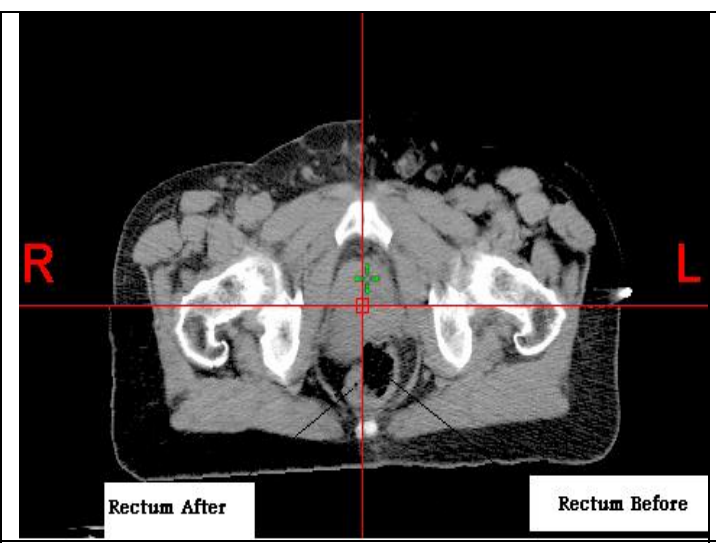

Fig ( 4 ) : Displacement of Prostate due to Rectum Volume change (image Fusion )

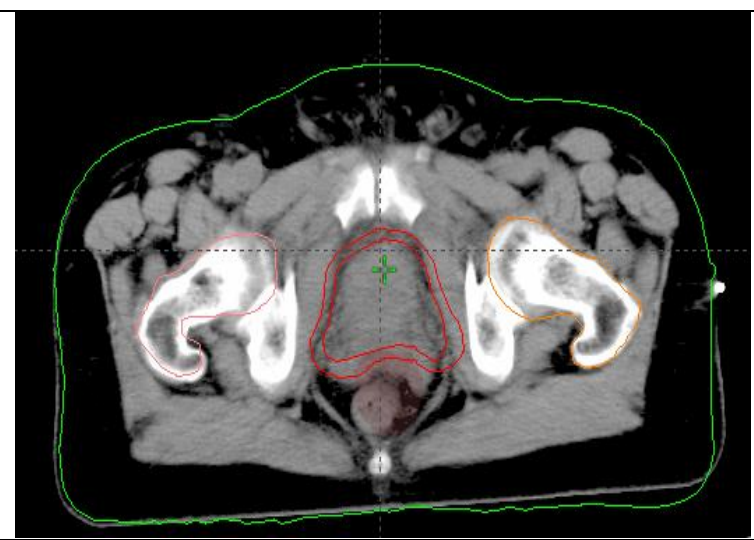

Fig (5 ) : Displacement of PTV and CTV for Same Patient.

- As show in Fig (4) ) the displacement in PTV and CTV in decrease in local control in tumor due to decrease in average volume was received optimum dose $95 \%$.

- According to multi studies done for coverage of PTV- there is no ideal coverage but coverage may be change but should be not, less than $95 \%$ from volume not received not less $95 \%$ from prescribed dose.

- In comparison with many study for PTV for Prostate displacement due effect of bladder and rectum varied within $5 \mathrm{~mm}$ to $12 \mathrm{~mm}$, but the current study the variation of displacement in average $5 \mathrm{~mm}$. ( as shown in Fig . 5).

Fig . 5 illustrated the PTV , CTV and Rectum volume from previous CT done for same patient with different condition.( tow different CT scans fusion for the Same Patient )

- This displacement increase the Rectum dose (restricted the prescribed dose for Prostate (reduce the local control of patient to control disease plus increase the risk factor for rectum damage.

- According this variation - the recommendation PTV should be included this value in addition the uncertainties values.

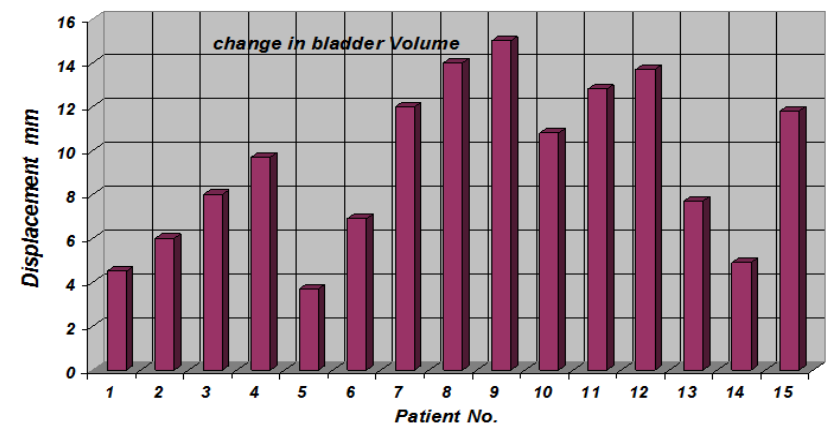

Fig.(6) : The Displacement in Bladder Volumes

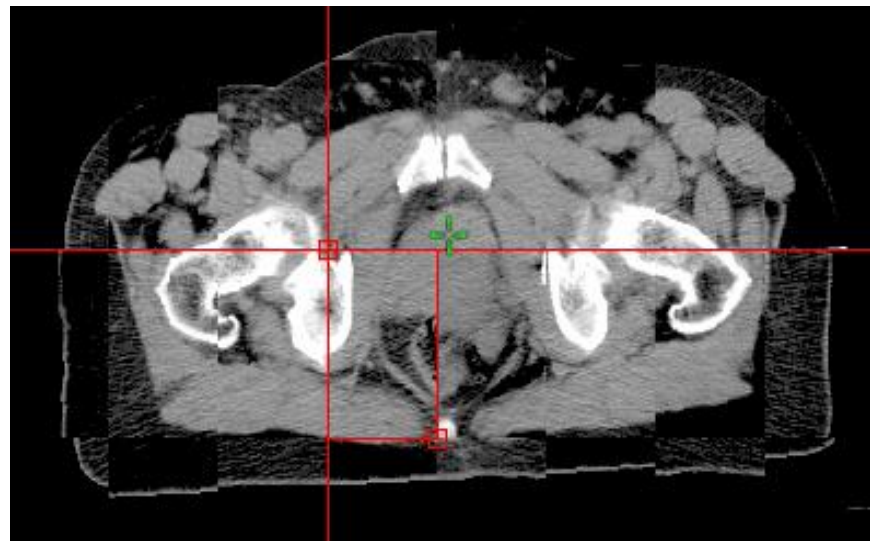

Fig.(7 ) : Fusion Image displayed the Change in Bladder Volume Two CT scans in different condition for the same patient 
-in Fig. (7);- as shown the shape of bladder change due to filling and emptying - according volume change toe volume of CTV and PTV displaced - change the dose cover target will decrease .

- According for target cover by optimum dose the local will affect .

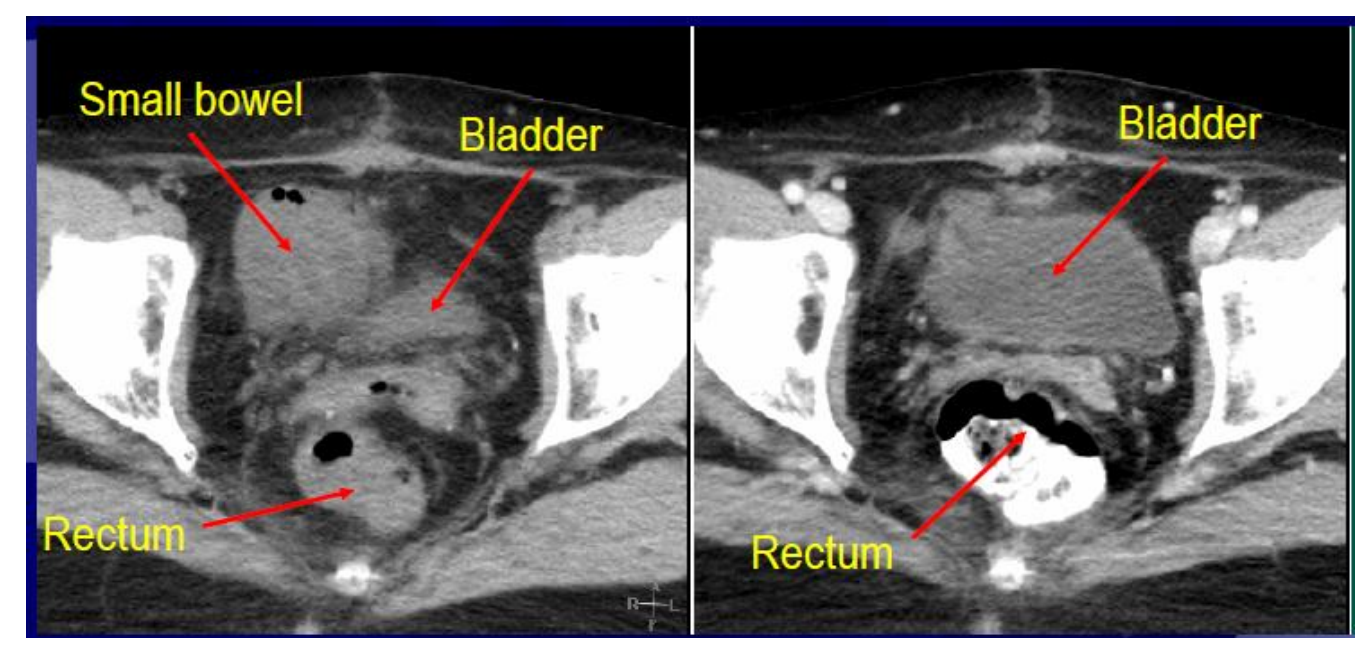

Fig (8) : Displacement due to Rectum and Bladder Volumes Changes

-As show Fig (8): illustrate the displacement in critical Volumes and PTV due to empty and filling the bladder and rectum.

- $\quad$ According study for displacement and margins for PTV about $9 \mathrm{~mm}$ for safety bladder and rectum dose tolerances.

- Good arrangements for planning is five fields size due to reduce the bladder and rectum dose in addition the Head of femurs within the tolerances of critical structures.

- $\quad$ In Fig (8) : illustrate the fusion between two image for different condition for imaging according empty and filling of bladder and rectum .

- Accruing my thesis can see the best condition the rectum should be empty to sure the PTV not displacement due to increase dose was received by critical like Bowel loop and Bladder.

- Bladder according condition of image empty or filling during the all fractions (all setting of treatment for Prostate patient) to reduce the dose to bladder as much as you can.

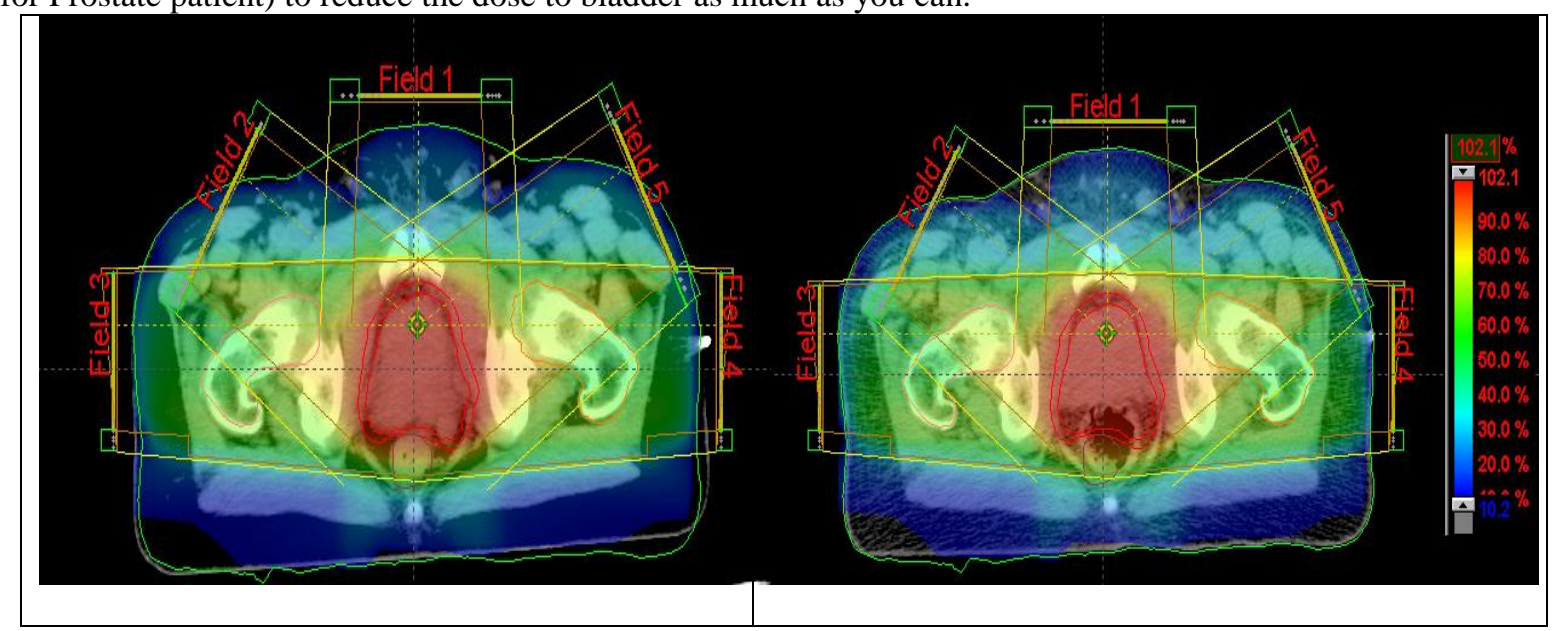

Fig. (9): Axial View for Rectum Volume variation the same patient

- As show in fig (9): the dose to rectum is increase due to change in volume and displacement of target PTV.

- $\quad$ This problem was solve by optimum margins about $9 \mathrm{~mm}$ in PTV and CTV .

In comparison with different studies were done the average margins about $12 \mathrm{~mm}$ in agreement with current study 


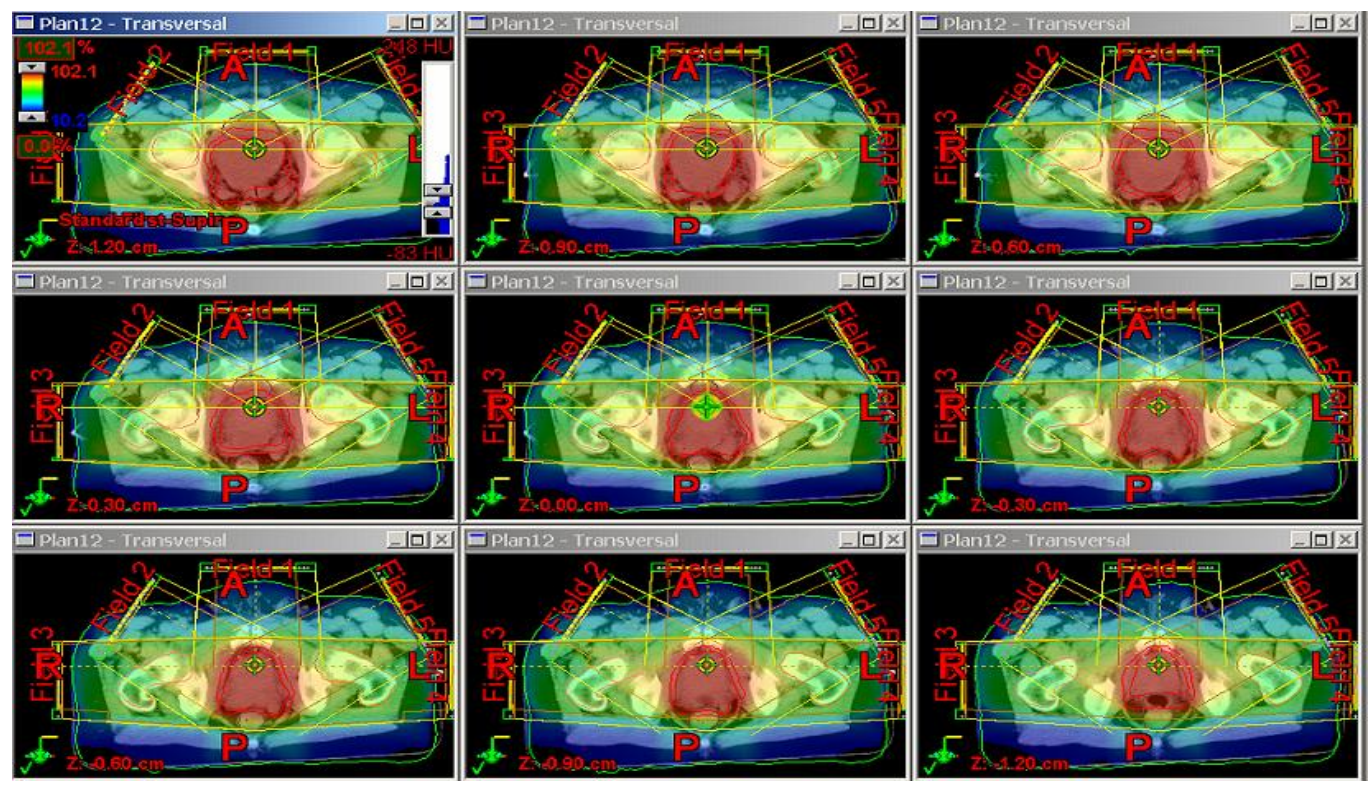

Fig (10) : Target evaluation for Rectum Displacement during different CTs

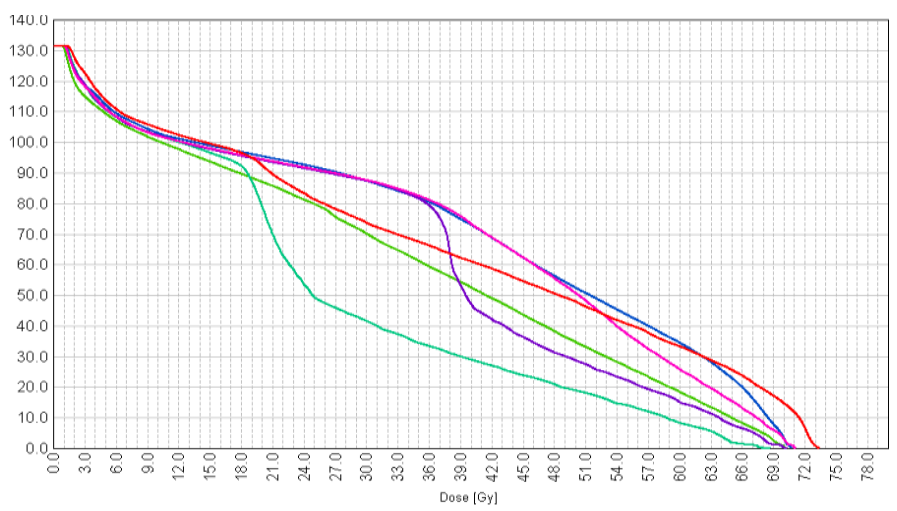

Fig.11.Different Dose volume histograms displays the different conditions of bladder for the same Patient

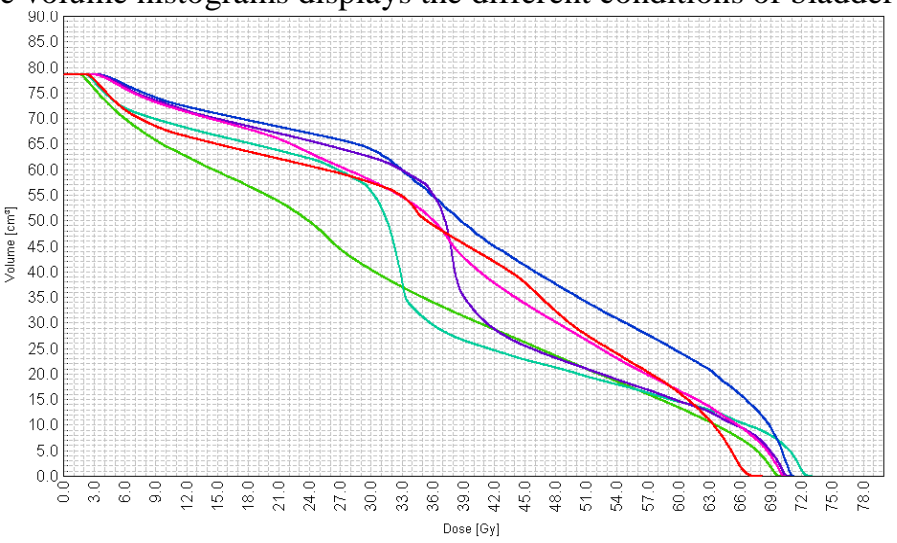

Fig.12. Different Dose volume histograms displays the different condition Rectum for the same patient

\section{Conclusions:}

Prostate displacement due effect of bladder and rectum varied within $5 \mathrm{~mm}$ to $12 \mathrm{~mm}$, the variation of displacement in average $5 \mathrm{~mm}$. Geometric Verification is Mandatory for all external beam radiotherapy. The geometric Verification Process must be carried out within a clearly structure, adhering to locally protocols. Each radiotherapy department should determine the verification protocols as planning margins required for their own practice. This because the frequency if imaging , the tolerances and action levels used, and the planning margins will vary according to local use of techniques, process, anatomical sites equipment and immobilization. 
Clinical implementation of geometric verification protocols should be co-coordinated a designated multi-professional team. Set- up errors have both systematic and random component, verification protocol are necessary to identify each component. Systematic error must be identified and minimized using correction protocols for every patient having a multifactor course of radiotherapy.

\section{References}

[1] Shen S, Duan J, Fiveash J B, Brezovich I A, Plant B A, Spencer S A, Popple R A, Pareek P N and Bonner J A Validation of target volume and position in respiratory gated CT planning and treatment Med. Phys. 30 3196-205, 2003.

[2] ICRU report 50: Prescribing, recording, and reporting photon beam therapy. Bethesda, MD: International Commission on Radiation Units and Measurements; 1993.

[3] ICRU report 62: Prescribing, recording, and reporting photon beam therapy (Supplement to ICRU report 50). Bethesda, MD: International Commission on Radiation Units and Measurements; 1999.

[4] Book Geometric Uncertainties in Radiotherapy - Defining the Planning Target Volume: The British Institute of Radiology; 2003.

[5] 5.Van den Heuvel F, Fugazzi J, Seppi E, Forman JD. Clinical application of a repositioning scheme, using gold markers and electronic portal imaging. Radiother Oncol;79:94-100, 2006.

[6] Van Elmpt WJC, Nijsten SMJJG, Mijnheer BJ, Minken AWH. Experimental verification of a portal dose prediction model. Med Phys;32:2805-18, 2005.

[7] Van Elmpt WJC, Nijsten SMJJG, Schiffeleers RFH, et al. A Monte Carlo based three-dimensional dose reconstruction method derived from portal dose images. Med Phys;33:2426-34, 2006.

[8] Van Esch A, Depuydt T, Huyskens DP. The use of an aSi-based EPID for routine absolute dosimetric pre-treatment verification of dynamic IMRT fields. Radiother Oncol;71:223-34, 2004.

[9] Stroom JC, de Boer HC, Huizenga H, et al. Inclusion of geometrical uncertainties in radiotherapy treatment planning by means of coverage probability. Int J Radiat Oncol Biol Phy.;43:905-919, 1999.

[10] Erridge SC, Seppenwoolde Y, Muller SH, et al. Portal imaging to assess set-up errors, tumor motion and tumor shrinkage during conformal radiotherapy of non-small cell lung cancer. Radiother Oncol;66:75-85,2003.

[11] Hurkmans CW, Remeijer P, Lebesque JV, Mijnheer BJ. Set-up verification using portal imaging; review of current clinical practice. Radiother Oncol;58:105-20, 2001.

[12] van Zijtveld M, Dirkx MLP, de Boer HCJ, Heijmen BJM. Dosimetric pre-treatment verification of IMRT using an EPID; clinical experience. Radiother Oncol;81:168-75, 2006.

[13] Van Zijtveld M, Dirkx MLP, de Boer HCJ, Heijmen BJM. 3D dos reconstruction for clinical evaluation of IMRT pretreatment verification with an EPID. Radiother Oncol;82:201-7,2007. 